\title{
FUENTES Y METODO PARA ESTIMAR \\ LA RENTA REGIONAL: SANTANDER, 1895-1930
}

PATRICIO PEREZ GONZALEZ

Universidad de Cantabria

\section{RESUMEN}

El ensayo consiste en una estimación de la renta regional por la via del producto, aplicado a la economia de Cantabria durante el primer tercio del siglo xx. Tras una in. troducción al modelo, se exponen los principales resultados, problemes e insuficiencias que encuentran soporte metodológico en el anexo.

\section{ABSTRACT}

This esany presents an estimation of regional income, for early 20th Century Cantabria. After a short introduction, the paper presents the major results. Methodological support is set forth in the annex.

\section{INTRODUCCION}

La historiografia económica cuantitativa ha progresado mucho en el proceso de estimación de la renta nacional ${ }^{1}$, si bien la estimación de las rentas provinciales y regionales está por hacer, como tuve ocasión de comprobar en mi tesis ${ }^{2}$. En consecuencia, he procurado elaborar una guía que sea de utilidad para historiadores y economistas que trabajen en temas regionales, en base a

' Carreras (1984, 1985a y 1989a), Prados (1988), Coll (1989). Véanse, ademais: Martin Aceña y Prados (1985), Sanchez-Albornoz (1985), Nadal, Carreras y Sudrià (1987) y Carreras (1989b).

2 Nadal y Carreras (1990) ha significado un gran avance. 
las fuentes y el método empleados para estimar el Producto Interior Bruto (PIB) de la provincia de Santander durante el primer tercio del siglo $\mathrm{xx}^{3}$. A tal fin, he optado por hacer un análisis en términos de Contabilidad Nacional por sus innegables ventajas: $1 .{ }^{a}$ ) considerar la economía regional como un subsistema del sistema representado por la economía española; $2 .^{a}$ ) aprovechar estimaciones que, siendo output de algunos procesos, constituyen input para otros; $3 .{ }^{2}$ ) llevar a cabo contrastes sucesivos que pongan a prueba la coherencia interna del modelo; y 4.) investigar las relaciones con otros subsistemas provinciales y regionales. El cálculo se hace a precios de mercado, que posteriormente es expresado a precios de 1913 tras ser deflactado con el indice de la Comisión del Patrón-Oro ${ }^{4}$.

Bajo el enfoque del producto, la renta se calcula como suma de los valores añadidos por los subsectores y ramos de actividad, hasta el nivel de desagregación que las fuentes permitan. La preferencia por esta via no excluye el recurso a la vía del ingreso o la del gasto cuando resulta necesario '. En principio, respetamos los datos de las fuentes originales -a nivel regional no existen más estadísticas que a nivel nacional - salvo que haya contradicción entre ellas o resulten incompatibles con el modelo; sölo entonces se procede a rectificarlas, no sin antes justificar los motivos y el procedimiento.

Bajo la óptica del análisis en términos de Tablas Input-Output (TIO), el valor añadido bruto (VAB) se puede calcular, directamente, como sumatorio de retribuciones a los factores de producción o, indirectamente, deduciendo del valor de producción a precios de salida de fábrica (VPF) el valor de los inputs intermedios. En general, se ha elegido una vía semidirecta, consistente en estimar el VAB como proporción del VPF en base a las TIO de 1958, las primeras realmente fiables. El procedimiento, insatisfactorio, se justifica, exclusivamente, por la imposibilidad de acometer la estimación directa en el estadio actual de nuestros conocimientos, pues se requeriria disponer de la contabilidad regional, cuando la contabilidad nacional sólo estuvo disponible bastantes años después. Sin embargo, la solución no puede ser despreciar los datos, por poco fiables, sino tomarlos con precaución.

El método tiene precedentes, dentro y fuera de nuestro pais. Hipótesis semejantes las han utilizado Schwartz, Carreras y Prados, entre otros, por no hablar del Banco Bilbao Vizcaya ${ }^{6}$. En el exterior, Chenery y Syrquin extrapolan

3 Véase mi tesis (Pèrez, 1991), que publicará próximamente la Cámara de Comercio, Industria y Navegación de Cantabria.

- Ministerio de Hacienda (1960).

3 Rojo (1979), Muñoz Cidad (1991).

6 Schwartz (1977), Carreras (1984), Prados (1988), Molinas y Prados (1988), BBV y FIES. 
la estructura del PIB en 1964 al periodo 1950-1970. Gerschenkron va aún más lejos cuando aplica el coeficiente VAB/VPF de la industria de Estados Unidos, en 1899, a la italiana de 1881-1913 por falta de estadísticas para estimarlos directamente 7 . Es obvio que tanto la escasez de datos como las dudas que plantea el proceso de ponderación disminuyen el valor de los cálculos. No obstante, el método sirve al propósito que se persigue: estimación directa del VPF, estimación semidirecta del VAB y estimación del cambio estructural. Además, no hay que exagerar las objeciones a la utilización de coeficientes fijos, pues «el cambio de estructura del PIB global vendrá reflejado por el distinto peso que los indicadores van prestando a las ramas sobre las que se proyectan, a medida que nos movemos hacia atrás» ${ }^{8}$. Con objeto de facilitar la tarea, hemos hecho un análisis de corte transversal centrado en 1895, 1904, 1913, 1922 y 1930. Las técnicas shift-share simplifican el problema de estimación, si previamente se ha definido la relación entre patrones de corte trans. versal y series temporales (sugerimos elaborar índices de acumulación de capital, producción agraria y consumo de energia).

\section{CUADRO 1}

Valor añadido, por sectores (a precios de 1913, miles ptas.)

\begin{tabular}{lrrrrr}
\hline SECTOR & 1895 & 1904 & 1913 & 1922 & \multicolumn{1}{c}{1930} \\
\hline Agrario $\ldots \ldots \ldots \ldots \ldots \ldots \ldots \ldots \ldots$ & 82.067 & 65.518 & 80.086 & 124.761 & 129.419 \\
Industria $\ldots \ldots \ldots \ldots \ldots \ldots \ldots \ldots$ & 24.864 & 29.699 & 37.881 & 42.270 & 59.139 \\
Servicios $\ldots \ldots \ldots \ldots \ldots \ldots \ldots \ldots \ldots$ & 59.085 & 49.834 & 62.421 & 85.512 & 108.465 \\
Total, ptas. constantes $\ldots \ldots \ldots \ldots \ldots$ & 166.016 & 145.051 & 180.388 & 252.553 & 297.023 \\
Total, ptas. corrientes $\ldots \ldots \ldots \ldots$. & 113.373 & 124.884 & 180.388 & 398.626 & 498.190
\end{tabular}

(Por ciento del total)

\begin{tabular}{llllll} 
Agrario $\ldots \ldots \ldots \ldots \ldots \ldots \ldots \ldots \ldots$ & 49,4 & 45,2 & 44,4 & 49,4 & 43,6 \\
Industria $\ldots \ldots \ldots \ldots \ldots \ldots \ldots \ldots$ & 15,0 & 20,5 & 21,0 & 16,7 & 19,9 \\
Servicios $\ldots \ldots \ldots \ldots \ldots \ldots \ldots \ldots \ldots$ & 35,6 & 34,4 & 34,6 & 33,9 & 36,5 \\
\hline
\end{tabular}

FUENTE: Pérez (1991).

'Chenery y Syrquin (1978), Gerschenkron (1968).

${ }^{8}$ Schwartz (1977), p. 492. 


\section{PRINCIPALES RESULTADOS}

\subsection{DINAMICA SECTORLAL Y CAMBIO DE ESTRUCTURA}

A finales del siglo xIx el sector agrario generaba la mitad de la renta provincial y los servicios más de un tercio. En cambio, la industria - que debia su importancia a los bienes de consumo- contribuía en proporción harto modesta (cuadro 1). Las fuentes manejadas y el sistema de cortes transversales contribuyen a profundizar una crisis finisecular $(-1,5$ por ciento) en tasa anual, cuadro 2) que sólo la industria pudo sortear $(+2$ por ciento), amparada en el buen momento de la mineria férrica y el comienzo de actividades en la siderurgia. El sector más perjudicado fue el primario ( $-2,5$ por ciento), a causa de la crisis agropecuaria, mientras que los servicios $(-1,9$ por ciento) tuvieron su crisis particular en la pérdida de los mercados coloniales protegidos.

\section{CUADRO 2}

Crecimiento del producto regional (Tasa anual, en porcentaje)

\begin{tabular}{ccccccc}
\hline SECTOR & $1895-1904$ & $1904-1913$ & $1913-1922$ & $1922-1930$ & $1895-1930$ \\
\hline & & & & & \\
Agrario $\ldots \ldots \ldots \ldots \ldots \ldots \ldots$ & $-2,5$ & 2,3 & 5,0 & 0,5 & 1,3 \\
Industria $\ldots \ldots \ldots \ldots \ldots \ldots$ & 2,0 & 2,7 & 1,2 & 4,3 & 2,5 \\
Servicios $\ldots \ldots \ldots \ldots \ldots \ldots \ldots$ & $-1,9$ & 2,5 & 3,6 & 3,0 & 1,8 \\
Total ................... & $-1,5$ & 2,5 & 3,8 & 2,0 & 1,7 \\
Deflactor del PIB ........ & 2,6 & 1,7 & 5,2 & 0,8 & 2,6 \\
\hline
\end{tabular}

Fuente: Pérez (1991).

A la crisis sigue un periodo de recuperación, entre 1904 y 1913, cuyo peso recae, a partes iguales, sobre las ramas agricola y ganadera del sector primario $(+2,3$ por ciento). También el secundario mantuvo una tendencia de crecimiento sostenido $(+2,7$ por ciento). Las mayores aportaciones proceden de la industria química, que coincidió en su despegue con la eléctrica y la de transformados metálicos. Si a esto añadimos el buen comportamiento de los servicios $(+2,5$ por ciento), auspiciado por la recuperación del comercio interior, el resultado no puede extrañar; a pesar de lo cual, el indice de producción se sitúa en 1913 en el nivel 109, en base 1895-100. 
Coincidiendo con la guerra y posguerra europeas se produce un cambio en las pautas de desarrollo regional, pues el aumento de la tasa de crecimiento general ( $+3,8$ por ciento) encubre grandes diferencias entre sectores. El mayor dinamismo corresponde al agrario ( +5 por ciento), que tiene, de nuevo, en la ganadería su ramo más activo. Durante estos años tuvo lugar una importante recuperación de la cabaña vacuna, acompañada por una notable selección de razas y la progresiva transformación de la explotación extensiva en intensiva. También los servicios salieron fortalecidos ( +3 por ciento), gracias a la linea de recuperación del comercio y el excelente comportamiento del subsector financiero. En cambio, el crecimiento industrial $(+1,2$ por ciento) fue decepcionante. La base de la industria pesada habia sido la mineria, cuyo producto quedó reducido a la tercera parte a raiz de la Gran Guerra; por contra, hubo aumentos significativos en los ramos químico, eléctrico y metalúrgico. Por lo que se refiere a las manufacturas, durante los primeros años veinte tuvo lugar el despegue de la industria agroalimentaria de base ganadera y la crisis de la textil. El resultado neto de todas esas tendencias es una reducción de más de dos puntos en la aportación de la industria al PIB (cuadro 1).

\section{CUADRO 3}

Productividad de la mano de obra (VAB en ptas. a precios de 1913)

\begin{tabular}{ccccccr}
\hline SECTOR & 1895 & 1904 & 1913 & 1922 & 1930 \\
\hline & & & & & & \\
Agrario $\ldots \ldots \ldots \ldots \ldots \ldots \ldots \ldots \ldots \ldots \ldots \ldots \ldots \ldots \ldots \ldots$ & 1.134 & 1.106 & 1.455 & 2.512 & 2.886 \\
Industria $\ldots \ldots \ldots \ldots \ldots \ldots \ldots \ldots \ldots \ldots \ldots \ldots \ldots \ldots \ldots$ & 1.793 & 1.984 & 2.472 & 1.655 & 1.531 \\
Servicios $\ldots \ldots \ldots \ldots \ldots \ldots \ldots \ldots \ldots \ldots \ldots \ldots \ldots \ldots \ldots$ & 2.963 & 2.521 & 2.798 & 3.281 & 3.334 \\
Promedio $\ldots \ldots \ldots \ldots \ldots \ldots \ldots \ldots \ldots \ldots \ldots \ldots \ldots \ldots \ldots$ & 1.563 & 1.544 & 1.946 & 2.494 & 2.560 \\
Industria (corregida) $\ldots \ldots \ldots \ldots \ldots \ldots \ldots \ldots \ldots \ldots \ldots \ldots$ & n.d. & 2.127 & 2.821 & 2.182 & 3.132
\end{tabular}

FúenTE: Pérez (1991).

A principios de los treinta la participación agraria en el PIB se reduce aún más, si bien la reducción responde no tanto a una crisis como a una reestructuracion, que le permite liberar una parte importante de la fuerza de trabajo sin reducir el producto agrario ( $+0,5$ por ciento). En este proceso, la ganadería ve reforzado su papel como eje aglutinante de un sector agrario que ha tendido múltiples puentes con el industrial.

En el corte de 1930 el producto industrial procede, casi a partes iguales, 
de la industria pesada (minería y electricidad incluidas) y la ligera. Sin embargo, la mayor aportación individual corre a cargo de la alimentación, que tiene en la producción de leche industrializada, chocolates, cuero, conservas de pescado, etc., sus mejores exponentes. Entre los ramos destacados de la industria pesada figura, por derecho propio, el eléctrico (cuadro 5). Asimismo hay que destacar la recuperación de la minería de cinc y la consolidación de la química. La confluencia de todas esas tendencias hizo posible que se alcanzaran las mayores cotas de crecimiento en la industria ( $+4,3$ por ciento), un sector que a comienzos de los treinta aportaba el 20 por ciento del PIB. En cuanto a los servicios, crecen a una tasa moderada ( +3 por ciento), como consecuencia de la coincidencia de diversas circunstancias. De una parte, el aumento de la población y su creciente urbanización favorecieron el desarrollo de los servicios relacionados con el consumo. De otra, la industria tiró de los servicios para la producción: profesiones liberales, banca y transportes. La consolidación de la estructura del Estado, en fin, tuvo como secuela el crecimiento del sector público. En 1930 el sector terciario aportaba algo más de un tercio del PIB.

\section{CUADRO 4}

Evolución de la renta per cápita (Ptas. constantes de 1913)

\begin{tabular}{ccccccc}
\hline \multirow{2}{*}{ Año } & & $\begin{array}{c}\text { Renta total } \\
\text { (miles ptas.) }\end{array}$ & $\begin{array}{c}\text { Pablación } \\
\text { total }\end{array}$ & $\begin{array}{c}\text { Renta } \\
\text { per cápita }\end{array}$ & $\begin{array}{c}\text { Aluarez } \\
\text { Llano }\end{array}$ & $\begin{array}{c}\text { ESPANA } \\
\text { Renta } \\
\text { per cápita }\end{array}$ \\
\hline $1895 \ldots \ldots \ldots \ldots \ldots \ldots \ldots \ldots$ & 166.016 & 263.339 & 630,4 & n.d. & n.d. \\
$1901 \ldots \ldots \ldots \ldots \ldots \ldots \ldots \ldots \ldots$ & 158.723 & 278.551 & 569,8 & 888,3 & 561,4 \\
$1904 \ldots \ldots \ldots \ldots \ldots \ldots \ldots \ldots \ldots$ & 145.051 & 286.484 & 506,3 & n.d. & 586,9 \\
$1913 \ldots \ldots \ldots \ldots \ldots \ldots \ldots \ldots \ldots$ & 180.388 & 310.168 & 581,6 & n.d. & 644,0 \\
$1922 \ldots \ldots \ldots \ldots \ldots \ldots \ldots \ldots \ldots$ & 252.553 & 334.660 & 754,7 & 972,9 & 739,4 \\
$1930 \ldots \ldots \ldots \ldots \ldots \ldots \ldots \ldots \ldots$ & 297.023 & 364.147 & 815,7 & 863,1 & 804,8 \\
\hline
\end{tabular}

Fuente: Alcaide (1976), Alvarez (1986), Pérez (1991).

\subsection{CRECIMIENTO Y ATRASO DE LA ECONOMIA MONTAÑESA}

Sin embargo, la tasa de crecimiento entre 1922 y 1930 ( +2 por ciento) es la más baja desde la crisis finisecular. Las diferencias intersectoriales de productividad sugieren que la asignación de recursos no ha sido eficiente, toda 
vez que la productividad media de la industria en 1922 y 1930 llega a ser poco más de la mitad que en agricultura y servicios.

\section{CUADRO 5}

$V A B$, por ramos de producción (A precios de 1913, miles ptas.)

\begin{tabular}{|c|c|c|c|c|c|c|}
\hline$C O D$ & RAMOS & 1895 & 1904 & 1913 & 1922 & 1930 \\
\hline Al & Agricultura & 10.862 & 9.978 & 15.727 & 19.570 & 12.704 \\
\hline A2 & Ganaderia & 66.410 & 44.305 & 50.375 & 80.541 & 98.421 \\
\hline Al & Pastos y montes. & 5.282 & 8.147 & 10.621 & 17.971 & 12.912 \\
\hline A3 & 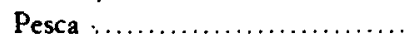 & 1.313 & 3.088 & 3.333 & 6.679 & 5.382 \\
\hline I1 & Combustibles sólidos & 6 & 4 & 52 & 143 & 150 \\
\hline 12 & Petróleo y gas natural ............ & 262 & 267 & 176 & 301 & 275 \\
\hline I4 & Energía eléctrica ...... & 238 & 726 & 1.563 & 3.546 & 6.918 \\
\hline II & Mineria metálica & 3.844 & 8.173 & 9.253 & 2.853 & 6.279 \\
\hline Il & Siderurgia ....... & - & 1.310 & 1.658 & 1.649 & 3.189 \\
\hline II & Mineria no-metálica .... & 1 & 11 & 13 & 983 & 1.147 \\
\hline 13 & Transformación minerales & 575 & 761 & 1.253 & 1.329 & 2.029 \\
\hline 12 & Industria quimica...$\ldots \ldots$ & 77 & 394 & 3.829 & 7.535 & 8.117 \\
\hline II & Productos metálicos & 659 & 1.000 & 938 & 1.933 & 1.441 \\
\hline 15 & Alimentación ........ & 13.420 & 11.713 & 12.434 & 14.874 & 20.332 \\
\hline Is & Textil $\ldots \ldots \ldots$ & 2.258 & 1.660 & 2.222 & 1.384 & 2.451 \\
\hline Is & Cuero y calzado ...... & 261 & 99 & 230 & 289 & 972 \\
\hline I5 & Madera y corcho $\ldots \ldots \ldots \ldots \ldots$ & 10 & 68 & 307 & 1.087 & 822 \\
\hline I5 & Papel y artes graficas $\ldots \ldots \ldots \ldots$ & 0 & 48 & 99 & 82 & 190 \\
\hline I6 & Construcción ................... & 3.253 & 3.465 & 3.854 & 4.282 & 4.827 \\
\hline S1 & Comercio...$\ldots \ldots \ldots$ & 26.564 & 22.711 & 28.414 & 40.304 & 51.941 \\
\hline S1 & Hosteleria ........ & 11.001 & 9.406 & 11.768 & 16.692 & 21.511 \\
\hline Si & Transportes ..... & 11.297 & 7.079 & 9.281 & 8.245 & 9.215 \\
\hline S2 & Banca y seguros & 2.072 & 2.163 & 3.606 & 8.554 & 9.440 \\
\hline S3 & Propiedad de viviendas .......... & 101 & 164 & 269 & 440 & 682 \\
\hline \multirow[t]{2}{*}{ S4 } & Otros servicios $\ldots \ldots \ldots \ldots \ldots \ldots$ & 8.050 & 8.311 & 9.083 & 11.287 & 15.676 \\
\hline & TOTAL & 56.016 & 145.051 & 180.388 & 252.553 & 297.023 \\
\hline
\end{tabular}

FUENTE: Anexo.

¿Cómo explicar la caida de la productividad industrial? Existen dos tipos de factores, unos formales y otros de fondo. Entre los primeros cabe citar las fuentes y el sistema de cortes transversales, y entre los segundos, la caida del ahorro, la contracción de la inversión y los cambios en la composición del producto. En el marco de una política de autoabastecimiento del mercado nacio- 
nal y crecimiento de la demanda externa, «las nuevas inversiones, ante las dificultades de importación de bienes de equipo, se basan en la utilización de mano de obra excedentaria» 9 .

\subsection{EVOLUCIÓN DE LA RENTA REGIONAL}

La tasa de crecimiento global entre 1904 y 1930 fue de 2,8 por ciento, aunque si se contabiliza el período completo se reduce a 1,7 por ciento. El cociente entre renta y población devuelve una serie coherente de renta per capita. Al comparar nuestra estimación de la renta per capita en Cantabria con la de Alcaide para España ${ }^{10}$, se ve que las mayores discrepancias entre ambas se sitúan en los primeros cortes (cuadro 4). El valor inicial de su serie, que empieza en 1901 (561,4 ptas./hab.) coincide en la muestra, aproximadamente, con el resultado de interpolar entre 1895 y 1904 ( 569,8 ptas./hab.).

El crecimiento parece haber sido bastante más intenso en la provincia de Santander que en el conjunto de España entre 1904 y 1930, tal vez porque también fue más profunda la crisis precedente. La serie de Alcaide denota sólo lentificación del crecimiento; la de Carreras (1989a), por el contrario, detecta bien la caida de la renta entre 1904 y 1907. Durante ese largo cuarto de siglo, la renta per cápita de Santander crece a tasa del 1,85 por ciento anual, mientras que la española lo hace al 1,22 por ciento. Mucho más importantes son las diferencias con la estimación de Alvarez Llano (1986).

En el Anexo se hace un análisis de las fuentes y el método, tras agrupar las actividades en veinticinco ramas precedidas de un código identificativo (cuadro 5), de acuerdo con la Clasificación Nacional de Actividades Económicas de 1974 con dos dígitos significativos.

\section{A NEXO}

\section{SECTOR AGRARIO}

\subsection{SUbSeCtor agricola.Forestal [A1]}

En este sector seguimos de cerca los trabajos del Grupo de Estudios de Historia Rural (GEHR). En la estimación del VAB, además de las fuentes habi-

\footnotetext{
- Roldán y Garcia Delgado (1973), p. 23.

10 Alcaide (1976), p. 1146.
} 
tuales, hemos tenido la fortuna de manejar memorias inéditas, asi como aislar algunas constantes de la explotación agraria. Las fuentes son diversas, aunque la primaria fuera siempre la misma. La Sección Agronómica Provincial elaboraba, anualmente, la Memoria Reglamentaria por duplicado: un ejemplar se enviaba al Ministerio de Agricultura y otro quedaba en la provincia. Dichos informes, en general bastante concisos y escritos con estilo sencillo, constituyen la base de los estudios elaborados por la Junta Consultiva Agronómica (JCA); posteriormente se publicaban, en forma de Avances, agrupados por regiones agricolas. Aunque la calidad no sea homogénea, su nivel es bueno: además de los datos propiamente estadísticos, contienen una detallada referencia de las condiciones de explotación. La Sección Agronómica facilitaba, asimismo, información a la Cámara de Comercio, Industria y Navegación, que la incluia en sus Memorias anuales. La consulta de esta fuente no resulta estéril, porque los Avances de la JCA no cubren todos los años y no se conserva copia de todas la Memorias Reglamentarias. A partir de 1927 se reorganizaron los servicios de recogida de los datos de las producciones agrícolas, al tiempo que se ampliaba el número de cultivos: ésta sería la base de los Anuarios Estadisticos de Producciones Agricolas (AEPA).

En general, todas las fuentes agricolas incluyen superficies, producto y valor de producción ${ }^{11}$. Las estadisticas correspondientes a los primeros años son las más imprecisas. En primer lugar, porque «junto a sus probables imperfecciones (...) están las enormes lagunas: sólo se ocupaban del sistema cereal, la vid y el olivo.... 12. En segundo lugar, por la costumbre de la Junta de dedicar la Memoria de cada año a un cultivo distinto, por lo cual hay que ampliar el período de referencia si se quiere tener una estadistica completa.

La consideración de las superficies y producciones agrarias invita a la reflexión. A tenor del Avance de 1923, la extensión de la superficie agraria en los primeros años veinte coincidió con la intensificación de los cultivos. La mejora afectó, sobre todo, a la producción de montes, dehesas y pastos. Sin embargo, esa partida aparece distorsionada hasta el punto de alterar la composición del producto. La anomalía resulta especialmente notoria porque contradice la Estadistica de Producción de los Montes de Utilidad Pública (EPMUP) ${ }^{13}$. A la vista de estas circunstancias, hacemos una estimación alternativa de 1922 en

11 Sección Agronómica Provincial (1889, 1890, 1900, 1908), Junta Consultiva Agronómica (1891), Ministerio de Agricultura, Industria, Comercio y Obras Públicas (1902, 1905a), Ministerio de Fomento (1915, 1913, 1914a, 1923), Ministerio de Agricultura, Industria y Comercio (1932), Cámara de Comercio (1913, 1914), GEHR (1985).

12 GEHR (1983), p. 190.

13 GEHR (19836), p. 278, EPMUP. 
los siguientes términos: $\left.1 .^{\circ}\right)$ Estimación del producto de praderas naturales, de acuerdo con la hipótesis de superficie equivalente a la media de 1913 y 1931 , y el rendimiento de este último año. $2^{\circ}$ ) Valoración de los montes a tenor de la EPMUP, dando por bueno el resto de las producciones porque los rendimientos guardan cierta coherencia con los de otros años. 3.9) Corrección de posibles errores motivados por causas, aparentemente, tipográficas. El proceso de reestimación alcanza también a las praderas naturales, en 1913, para lo cual se ajusta el rendimiento de ese año regresando los valores reales de los demás años.

\subsection{SUBSECTOR GANADERO [A2]}

El método de estimación elegido se basa en el cálculo de coeficientes específicos para cada tipo de ganado. Aplicados al número de animales, se consiguen aproximaciones a las producciones en las fechas correspondientes a cada corte. El concepto de producto ganadero se limita a la producción de carne, leche y lana. Los censos de ganados, sobre todo los generales, son una fuente insustituible; los mejores son los de 1865, 1891, 1917, 1929 y 1933 14. Los resúmenes hechos por la JCA, en base a las memorias redactadas por los ingenieros del servicio agronómico, son del mayor interés. La incorporación de monografias provinciales resulta muy acertada, pues, además de la información propiamente estadística, contienen referencias a los aspectos zootécnicos, económicos y de fomento de las razas; en adelante nos referiremos a ellos como Avance de 1891 y Estudio de $1917^{15}$. Además, existen otras fuentes complementarias como Prados y Pastos, Dirección General de Aduanas y Norte. También resulta provechoso consultar las memorias de la Sección Agronómica Provincial y la Junta Provincial de Abastos, pues contienen referencias que no siempre aparecen en las fuentes nacionales.

\subsubsection{Peso en vivo de la ganadería y precios de los productos}

Para pasar del número de cabezas al peso en vivo se utilizan pesos unitarios manejados por la JCA en el Estudio de 1917. El periodo más incierto corresponde a $1865-1900$, toda vez que la falta de otros censos hace recaer la

14 GEHR (1978) incluye los censos de 1865, 1891, 1905, 1907-1908, 1910-1918, 1921, 1925 y 1929. Por nuestra parte, incorporamos los de 1900, 1909, 1924 y 1933.

15 D. G. de Agricultura, Industria y Comercio (1892), Ministerio de Fomento (1923). 
carga de la prueba del movimiento convulsivo finisecular sobre el Avance de 1891 y la Memoria de 1900. A tenor de aquél, la crisis pecuaria debe venir de algún momento entre 1891 y 1900. Sin embargo, la Memoria de este año habla ya de florecimiento del ganado vacuno, base de la riqueza ganadera provincial 16 .

La inseguridad del censo de 1891 afecta al propio recuento. La Guía de Santander de ese mismo año lamenta que el Estado - a falta de datos para fijar los cupos de contribución por la riqueza pecuaria- los establece «a ojo de buen cubero, aumentando, por ejemplo, a los de 1865 un 25 por ciento...» 17. La comparación del censo de 1865 , actualizado con ese criterio, y el Avance de 1891 arroja una sombra de duda sobre su fiabilidad. Hemos supuesto que ambas estadisticas (la de 1891 y la de 1900) son correctas, pero que aquélla se retrotrae al segundo lustro de los ochenta, por cuanto la estimación de años intermedios se obtiene por interpolación.

Una vez estimada, la producción ganadera hay que valorarla. El AEPA sólo ofrece precios a partir de 1929. Para fechas anteriores hemos debido recurrir a la estimación directa, a partir de diversas fuentes ${ }^{18}$.

\subsubsection{Producción de carme, leche y lana}

Con la información recogida para el estudio de la ganaderia, se ha estimado la producción de carne. Primero se compara el número de reses sacrificadas de cada especie con los correspondientes censos. Después se calculan -en base a los datos del AEPA de 1930 - los pesos en vivo de las reses sacrificadas y se comparan con el peso total de la cabaña. Finalmente se aplican coeficientes de rendimientos fijos - deducidos del Estudio de la ganadería, de 1917 - a las cifras de peso en vivo total, para estimar los pesos en canal a partir de una mix fija. La aplicación de rendimientos constantes se justifica por la escasa variación apreciada en los pesos unitarios ${ }^{19}$. Los ganaderos montañeses destinaban para carne, anualmente, una parte fija de su cabaña que oscila entre el 13 y el 14 por ciento del peso en vivo. Aunque la estructura de la demanda variara de un año a otro, la proporción era relativamente estable.

16 Sección Agronómica de Santander (1900).

17 Coll y Puig (1891). D. G. de Agricultura, Industria y Comercio (1892).

${ }_{18}$ Ministerio de Fomento: D. G. de Agriculcura, Industria y Comercio (varios años). Sección Agronómica de Santander (1900), Cámara de Comercio, Industria y Navegación de Santander (varios años) y Junta Provincial de Abastos de Santander (1927).

19 D. G. de Agricultura, Industria y Comercio (1892), Ministerio de Fomento (1920), Ministerio de Aricultura, Industria y Comercio (1932). 
La producción de leche es una variable aleatoria que depende del número de reses ordeñadas, su capacidad lechera y el régimen de explotación. Limitamos la discusión al ganado vacuno, pues representaba más del 99 por ciento del total. En Santander confluyeron a principios del siglo $\mathrm{xx}$ dos procesos complementarios. Uno, de sustitución de razas autóctonas por extranjeras, y otro, de progresiva implantación en la zona costera de la explotación intensiva del ganado vacuno. La confluencia de ambos procesos daría como resultado una mejora del rendimiento, que coincide en el tiempo con una expansión de la cabaña. Es difícil evaluar su intensidad, aunque - por el solo efecto de los cambios en el régimen de explotación - se ha cifrado en el 1,15 por ciento anual acumulativo. El cálculo se basa en las condiciones restrictivas que resultan de considerar la vaca constante, haciendo caso omiso de las ganancias inherentes a la mejora de las razas, que a no dudar existieron. De resultas de multiplicar el número de reses ordeñadas por su productividad, se llega a la producción de leche.

En cuanto a la producción de lana, se aplican coeficientes fijos tanto a la proporción de esquila (77por ciento) como al peso medio del vellón ( 2 kilos las reses adultas y setecientos gramos las crias).

\subsection{SUBSECTOR PESQUERO [A3]}

Las estadísticas correspondientes al primer tercio del siglo $\mathrm{xx}$ no empiezan hasta 1907. Al principio la metodología era escasamente homogénea, aunque después se fue normalizando ${ }^{20}$. Hemos estimado el volumen de capturas a partir de la ecuación de compensación: Capturas-Consumo en fresco+Destinado a conservas-Importación neta.

a) Consumo en fresco. En primer lugar, se ha estimado la estructura de la demanda para 1907, 1913, 1920 y 1930, como fechas más próximas a los cortes. A continuación comparamos el consumo de pescado en fresco con la población, de donde resulta el consumo per cápita. Conocida la población, es inmediato calcular la demanda interna.

b) Pescado para conservas. Para obtener un kilo de conservas se necesitan 1,69 de pescado, por término medio. Si suponemos que el coeficiente se mantiene fijo - y hemos estimado previamente la producción de conservas- podremos calcular la demanda de pescado por la industria conservera.

20 Ministerio de Marina (varios años), Alaejos Sanz (1922, 1931), Diputación Provincial de Santander (1929), Ministerio de Industria y Comercio (1933). 
c) Saldo comercial Desde el litoral se enviaba pescado fresco hacia el interior, si bien en las estadísticas de Norte no figura como partida independiente, al contrario que en las estadisticas de comercio exterior y de cabotaje, y las del Ferrocarril Cantábrico.

d) Volumen de capturas y valor de producción. La suma de la demanda de pescado para consumo en fresco y conservas, corregida por el saldo del comercio exterior, constituye una aproximación al volumen de capturas, cuya tendencia responde satisfactoriamente a los datos observados. El producto de capturas y precios - ya corregidos de las desviaciones entre precios estadísticos y reales de comercio exterior y cabotaje- nos da el valor de producción.

\section{SECTOR INDUSTRIAL}

\subsection{INDUSTRIA PESADA}

La principal fuente es la Estadística Minera y Metalúrgica (EMM). Publicada por el Consejo de Mineria, cubre el laboreo y el beneficio de los minerales. Entre otros aspectos de interés, contiene información sobre la producción (en toneladas y valor) y los factores empleados (mano de obra y fuerza motriz). En este contexto, el uso de otras fuentes se limita a cubrir posibles lagunas de la EMM, por dos motivos: ramas de actividad que, propiamente, no tienen cabida en aquélla y retrasos en la incorporación de algunas empresas cuya actividad cae de lleno dentro de la Estadística. Mención aparte merecen algunos trabajos de historiografia económica regional 21.

\subsubsection{Minerometalurgia [11]}

Existen algunas ramas que la EMM no recoge, como construcción naval y estampación en lata, que pueden considerarse subsidiarias de la actividad pesquero-conservera, con la que comparten fuentes. Para estimar la producción partimos de las referencias conocidas e indiciamos el valor desconocido en una variable asociada de la que haya suficiente información. La base para la provincia de Santander son dos excelentes memorias; en lo sucesivo nos referitemos a ellas como Memoria de 1904 y Estadística de 1909 22. Incluyen estadis-

21 Ortega (1986, 1990).

22 Ministerio de Agricultura, Industria, Comercio y Obras Püblicas (1905b). Ministerio de Fomento (1911). Se publicaron tambien para otras provincias, pero la calidad no es homogénea. 
ticas sobre número de empresas, empleo, equipos, proceso de fabricación y, en algunos casos, también producción final.

Usamos el valor de los equipos de pesca para estimar el de la construcción naval y, donde esta serie termina, la de capturas de pescado. Para el corte de 1930, la fuente obligada es el Ministerio de Industria y Comercio (1933). Puede estimarse la producción de envases a partir de la estructura de costes de la industria conservera.

\subsubsection{Industria quimica [I2]}

El primer corte en que la EMM da cuenta del beneficio de productos quimicos es 1922. La cuestión se reduce, entonces, a estimar la producción de los primeros cortes. La de 1904 se obtiene de las memorias antes señaladas, y puede intentarse una aproximación a la de 1895 contabilizando exclusivamente los artículos de consumo más tradicionales, puesto que las principales empresas químicas se constituyen con posterioridad. Finalmente, se ha estimado la producción de 1913 a partir de una función de demanda que relaciona los datos disponibles (de 1904, 1909, 1922 y 1930) con un indice que tome en cuenta el crecimiento demográfico (ilustrativo de la demanda interna) y el sal. do de la balanza comercial (exponente, a su vez, de la demanda externa). Puede hacerse una segunda aproximación a partir de las memorias de las compañias ferroviarias que daban servicio a las principales empresas; es el caso de Solvay y el Ferrocarril Cantábrico. La calidad de esta fuente es estimable, aunque no alcanza de lejos la de Norte. Todas las producciones han sido valora. das a precios de mercado.

Otro producto a tener en cuenta es el gas del alumbrado, que la EMM tampoco recoge hasta 1930. Utilizamos la Estadistica sobre Consumo de Gas ${ }^{23}$ para estimar su producción en 1904, 1913 y 1922, asignando a 1895 la producción fiscal de 1901.

\subsubsection{Cerámica, vidrio y cemento [13]}

Lo mismo que en siderometalurgia y química, la EMM no recoge las producciones anteriores al corte de 1922. En 1930, además de cemento se fabrica vidrio y productos cerámicos. Para cortes anteriores acudimos a la Estadistica

23 D. G. de Rentas Públicas (varios años). 
de $1909{ }^{24}$ y la demanda de viviendas, variable con la que esta industria está estrechamente correlacionada.

\subsection{INDUSTRIA ELECTRICA [I4]}

Empezamos reconstruyendo la serie de potencia instalada para estimar después la producción de energía, en virtud de la marcada correspondencia que existe entre ambas

\subsubsection{Potencia instalada}

La historiografia económica española ha identificado tres etapas en la evolución de la industria eléctrica durante el primer tercio del siglo xx. En Santander la primera se extiende desde 1892 hasta 1911. El final de esta etapa y el inicio de la segunda coincide con el comienzo de las actividades de Electra de Viesgo, la empresa a la que desde entonces estaria vinculado el sector eléctrico. La última abarca la Dictadura de Primo de Rivera y la II República. Las fuentes para la primera y tercera etapas son oficiales, si bien resulta provechoso cotejarlas con las memorias de las compañias eléctricas, que constituyen, por falta de estadisticas oficiales, la principal fuente durante la segunda fase ${ }^{25}$. La estadística del Instituto Geográfico y Estadístico, de 1923, se refiere a empresas que explotan centrales hidráulicas, pudiendo completarse sin más que añadir las centrales térmicas ${ }^{26}$. La infraestructura eléctrica anterior a la Guerra Civil estaba ya configurada, por cuyo motivo es de aplicación al último corte la estadística de la Cámara Oficial de Productores y Distribuidores de 1935.

\subsubsection{Consumo de energía y valor de producción}

A partir de las memorias de Electra de Viesgo deducimos las cifras de potencia y producción del periodo 1911-1928. Conocida la producción de 1913

24 Consejo de Mineria (varios años), Ministerio de Fomento (1911, 1930).

2) Ministerio de Agricultura, Industria, Comercio y Obras Públicas (1901). Cámara Oficial de Productores y Distribuidores de Electricidad (1935). D. G. de Rentas Públicas (varios años).

${ }^{26}$ Instituto Geografico y Estadistico (varios años). Ministerio de Fomento (1930). Cámara Oficial de Productores y Distribuidores de Electricidad (1935). D. G. de Rentas públicas (varios años). 
y 1922, es posible estimar la de 1904 aplicando a la potencia de este año el coeficiente de utilización de 1911; otro tanto se puede hacer con 1895. La producción de 1930 procede del Ministerio de Fomento. Para estimar el valor calculamos el precio medio del kilovatio-hora de Electra de Viesgo en 1913 y 1922. Seguidamente indiciamos en base a 1913 el precio del alumbrado público entre 1895 y 1904, y el precio medio nacional durante el periodo 1913 1930. El valor de producción total es la suma de los consumos de energía para alumbrado y usos industriales, ponderados con sus precios respectivos.

\subsection{INDUSTRIA MANUFACTURERA 27 [I5]}

\subsubsection{Fuentes de naturaleza fiscal}

La introducción del impuesto sobre consumos por la reforma de Villaverde aporta una fuente de gran riqueza estadística ${ }^{28}$. Asignamos a cada corte la producción del año correspondiente, salvo 1895, al que atribuimos las del primer año fiscal, y 1930, al que hemos asignado la de 1929. Asi se han estimado las producciones de achicoria, azúcar y bebidas alcohólicas; para la de vino remitimos a las fuentes agricolas.

\subsubsection{Estadisticas de comercio}

El sistema de producción es un circuito cerrado, como señala Gómez Mendoza (1984). Toda mercancia producida o importada tiene como contrapartida ésa u otra mercancia gastada o exportada. En articulos de exportación, la producción es igual al saldo de la balanza comercial, y en los destinados al mercado interior que incorporan materias primas importadas, la producción es igual a dicho saldo multiplicado por el coeficiente de conversión de materia prima en producto. Los coeficientes pueden deducirse de las Memorias sobre la industria fabril y las propias de algunas compañias ${ }^{29}$. Por este procedimiento se ha calculado la producción de harinas, textiles, cacao, café, tabaco, aceite, leche y derivados, cueros y pieles, pasta para sopas y galletas, etc.

27 Para más detalle, véase Pérez (1992).

28 D. G. de Aduanas: Producción y circulación de azúcares, achicoria, alcohol y cerveza (varios años).

29 Ministerio de Hacienda (1900). 


\subsubsection{Estadísticas demográficas}

Existen algunas objeciones a su empleo con fines predictivos de la producción por la calidad de las fuentes. Tras las premisas subyacen hipótesis restrictivas, como la permanencia de los hábitos de consumo y la constancia de la propensión marginal al consumo. La combinación de esta fuente con la anterior y la siguiente permite extender el cálculo por métodos indirectos a las industrias que trabajan tanto para el mercado interior como para el de exportación. La estimación se obtiene, entonces, a partir de la producción del año (o años) base, indiciada en una variable asociada a la evolución de la oferta y/o la demanda, que puede ser la población ocupada, la población total o el saldo de la balanza comercial. De esta forma se ha estimado la producción de algunas ramas alimentarias (chocolate, café, dulces, gaseosas y jarabes, agua mineral, galletas y pasta para sopas, tabaco, harina, productos lácteos y conservas de pescado), textiles, cuero y calzado, madera, y papel y artes gráficas.

\subsubsection{Estadisticas de producción y consumo}

Las más completas son la Memoria de 1904 y la Estadística de 1909 (vid supra). En la medida que corresponden a un momento próximo al centro del intervalo 1895.1930 , proyectamos tendencias centradas en torno a la media. Su principal inconveniente reside en «aceptar implicitamente que no tienen lugar cambios en el producto por trabajadors ${ }^{30}$, de ahi la conveniencia de aplicarlo a ramas muy concretas y utilizar más de una base de ponderación. Las Memorias de la Junta Provincial de Abastos se refieren a productos de consumo que pasan por el mercado, pero no hay que infravalorar su alcance por la importancia de tales articulos a principios de siglo. El estudio de Alaejos sobre pesca marítima y los trabajos de Carmona contienen referencias interesantes para las conservas de pescado; otro tanto cabe decir del AEPA y la industria agroalimentaria "'.

A veces los productos están valorados en la fuente, aunque lo más frecuente es que haya que estimar también el valor. En tales casos retenemos el precio medio de exportación - salvo que no lo hubiera, en cuyo caso se aplica el de importación- corregido con el correspondiente coeficiente de desviación, estimado por Tena (1985) y Prados (1986).

\footnotetext{
${ }^{30}$ Prados (1988), pp. 40 y 144. Schwartz (1977).

31 Junta Provincial de Abastos de Santander (1927), Alaejos (1922), Carmona (1990) y Ministerio de Economía Nacional $(1929,1930$ y 1933$)$.
} 


\subsection{Construcción y Obras Públicas [16, S3]}

No es posible utilizar el método de estimación propuesto por Tafunell por falta de información. En Santander carecemos de datos relativos a las pólizas de las compañias de seguro. En consecuencia, hacemos uso de la fuente fiscal, dado que «la puesta en vigor de los Registros Fiscales en las grandes ciudades como aplicación de la Ley de Ensanche de las poblaciones de 1892 (...) supuso una creciente erosión del fraudew ${ }^{32}$.

\subsubsection{Riqueza urbana y registro fiscal}

Contamos con dos series distintas de riqueza urbana. La primera comprende el periodo 1898-1907, aunque se puede retrotraer hasta 1895; la segunda arranca de 1903 y, tras un vacío que dura más de una década, cubre con algu. na discontinuidad el período 1915-1928. Carecemos de información acerca del periodo 1908-1914, pese a que (por ser de interregno entre ambas series) podria haber sido cubierto por cualquiera de ellas. La primera observación corresponde a 1879; se trata de una evaluación alzada de la riqueza urbana. La segunda atañe a la recaudación por este concepto en el ejercicio 1894-95. La obtención de la base imponible es inmediata, con sólo dividir la cuota entre el tipo de gravamen ${ }^{33}$. La información básica del periodo 1898.1907 son los valores liquidados por contribución urbana $y$, puesto que conocemos la riqueza imponible de algunos ejercicios, se aplica la tasa efectiva para estimar la base imponible de los demás años. Con las limitaciones ya sabidas, puede servir como aproximación a la riqueza inmobiliaria.

\subsubsection{Valor catastral y edificación}

Con el año 1903 empieza la segunda etapa, basada en el catastro de la riqueza urbana, que dio paso a un proceso de acercamiento de los valores catastrales a los de mercado ${ }^{34}$; la primera observación corresponde a 1915. En nuestra opinión, se deben actualizar las incorporaciones realizadas al registro

12 Tafunell (1988; 1989, pp. 254 y 265). Gómez Mendoza (1986). Instituto Geográfico y Estadistico: Nomenclátor...

33 D. G. de Contribuciones (1879). Ministerio de Hacienda (1897). Intervención General de la Administración del Estado (1909), pp. 443-451 y 566-588.

14 Ministerio de Hacienda (1918, 1921, 1922 y 1931$).$ 
cada año, pues, una vez fijados, los valores permanecian prácticamente inalterados.

En cuanto al periodo 1908-1914, en que las series se solapan, las valoraciones no coinciden porque ambas series miden el stock de construcción con criterios diferentes ¿Con cuál quedarse? Preferimos tomar como base la riqueza urbana y corregir el valor catastral en consecuencia. Como factor de corrección utilizamos el promedio de la relación entre los dos criterios en 1903 y 1907 (primero y último de los años comunes). En ambos casos la proporción es 2,3; es decir, que la riqueza urbana era, en promedio, más de dos veces superior al valor catastral ${ }^{35}$. La diferencia entre las valoraciones de años consecutivos equivale al incremento anual, que puede servir como aproximación al valor de lo edificado. Para que esto ocurra deben hacerse las correcciones relativas a reposición de edificios. Admitamos que la vida media de un edificio eran veinticinco años y que «el volumen de obra de los nuevos edificios superaba en un tercio al de los que sustituian» 36; incrementando en un 5,2 por ciento las cifras anteriores habremos corregido el efecto reposición.

La estimación de la riqueza catastral tiene otra finalidad. En tanto que aproximación a la riqueza urbana, sirve de base para calcular la renta imputada a la propiedad de viviendas, contabilizada como servicios diversos. En su estudio sobre la renta de la peninsula ibérica, Vandellós fija una «renta neta media del 4 por ciento» ${ }^{37}$ sobre el valor de la propiedad urbana. Al aplicar esa misma tasa a nuestra serie de riqueza urbana se obtiene la renta catastral en los años-corte.

\subsubsection{Obraspúblicas}

Los datos disponibles son escasos; en Santander los más completos corresponden al quinquenio 1923-1928. Forman parte de una publicación que da cuenta de las realizaciones durante la Dictadura de Primo de Rivera. La segunda fuente (ésta ya nacional) se refiere a las obras de construcción y conservación de carreteras por cuenta del Estado, entre 1912 y 1924, cuyos datos son coherentes con los de la fuente anterior. Por último, disponemos de información de la Cámara de Comercio, Industria y Navegación relativa a los trabajos

35 La regresión de los valores corregidos se ajusta satisfactoriamente a los valores observados (R $2-0,98$ ).

36 Tafunell (1989), p. 195.

37 Vandellós (1977), p. 121. 
realizados en el Puerto de Santander en el sexenio 1908-1913 38. Para las inversiones en infraestructuras ferroviarias, remitimos al Anuario de los Ferrocarriles Españoles.

Se ha supuesto que las obras públicas de los años veinte son representativas del último corte. Las estimaciones de los demás cortes puedan obtenerse a partir de aquéllas, indiciando en algunas variables significativas: las obras de infraestructura portuaria, en el movimiento de mercancías por los puertos de la provincia; las de infraestructura viaria, en el parque de vehículos; las ferroviarias, en el tráfico de mercancias; y las urbanas y de educación, en la población de los municipios mayores de cinco mil habitantes. Como alternativa (o complemento), puede consultarse la Estadística sobre el estado de las obras públicas en España.

\section{SECTOR SERVICIOS}

\subsection{Comercio, Transportes y Comunicaciones [S1]}

Empezamos analizando cada subsistema por separado. La integración de todos - comercio exterior, tráfico de cabotaje, transporte por ferrocarril y transporte por carretera- constituye la sintesis del sistema comercial ${ }^{39}$.

\subsubsection{Comercio marítimo}

En este apartado seguimos de cerca los trabajos de Prados (1982) y Frax (1981). La principal fuente es la Dirección General de Aduanas. Hasta 1920 las estadisticas de Comercio Exterior detallan los tráficos por puertos y provincias; después la publicación se interrumpe (a causa de los problemas que entraña su valoración) hasta 1930. En su defecto, recurrimos a las memorias de la Cámara de Comercio. Todas las series de exportación entre 1914 y 1920 están valoradas con los precios de 1913, por cuanto se han rectificado a la luz de las investigaciones de Prados y Tena.

De otro lado, aunque los métodos de valoración no fueron siempre los

38 Diputación Provincial de Santander (1929). Instituto Geográfico y Estadistico: Anuarios... (varios años). Cámara de Comercio, Industria y Navegación de Santander (1913).

39 D. G. de Aduanas: Estadistica general del comercio exterior y de cabotaje de España. Norte, Compañia del Ferrocarril Cantäbrico, Cámara de Comercio, Industria y Navegación de Santander, D. G. de Contribuciones: Estadistica del impuesto sobre los transportes... 
mismos, entre 1892 y 1920 las estadísticas del Comercio de Cabotaje están valoradas a precios de exportación; esto es, al coste promedio de producción. Por tal motivo le son de aplicación las rectificaciones propuestas para el comercio exterior, con la particularidad de que no hay más que una serie de coeficientes de rectificación, común para productos primarios y manufacturas. Las estadisticas dejaron de publicarse entre 1920 y 1936, por cuanto hay que recurrir, de nuevo, a las memorias de la Cámara de Comercio. Los datos del corte de 1922 corresponden a ese mismo ejercicio (los del Puerto de Santander) y a 1920 (los de otros puertos).

\subsubsection{Comercio por ferrocamil y mercado interior}

La base de nuestro método son los trabajos de Gómez Mendoza ${ }^{40}$, pues aunque el tráfico ferroviario no agota el comercio interior absorbe la fracción más importante. Consideramos el comercio de mercancias a través de Norte, no sölo porque canalizaba el volumen más importante de tráfico, sino porque el trazado de las otras compañias era esencialmente regional y, para nuestros fines, subsidiario de aquélla. Los Datos Estadísticos cubren, con algunas lagunas, el periodo 1874-1930 e identifican la posición deudora/acreedora de una determinada estación, pudiéndose obtener el saldo por simple comparación de los tonelajes llegados y expedidos. En cuanto a las cifras de träfico, son el resultado de sumar los movimientos correspondientes a todas las estaciones de Norte en la provincia. Las estadisticas distinguen entre tráfico local y combinado. El primero se refiere al tráfico entre estaciones explotadas exclusivamente por la compañia y el segundo al remitido a (o procedente de) otras compañias a través del empalme. En estaciones normales, la diferencia entre expediciones y llegadas equivale al saldo comercial; en las estaciones empalme el signo cambia, de modo que las mercancías expedidas puedan ser interpretadas como importaciones y las llegadas como exportaciones.

Se trata de estadísticas de peso. Todos los productos han sido valorados al precio medio de exportación, excepto las importaciones, que lo han sido al suyo propio. A tal fin, se aplican precios medios unitarios, que resultan de clasificar las series en 32 partidas.

. 40 Gómez Mendoza (1984, 1985, 1988). 


\subsubsection{Valor de producción}

El hecho de que en la estimación del producto comercial entren como in. puts el producto agrario e industrial obliga a hacer un examen recurrente; los datos de partida son los valores actualizados de los distintos subsistemas. Para estimar el valor del comercio y los transportes existe la posibilidad de apoyarse en los contemporáneos, que lo calculan a partir de una estructura-tipo. En su estimación de la renta española en la anteguerra, Vandellós cifra la industria de los transportes en un 23 por ciento de la producción industrial, comprendidos los productos de la pesca ${ }^{41}$. El porcentaje se distribuye entre transporte interior (ferrocarril y tranvia) y maritimo (cabotaje y exterior), en la proporción 65.35 por ciento. Entendemos que dicha proporción refleja la estructura particular de la economía española. Por tal motivo, podemos estimar la ratio de una provincia concreta, sin más que calcular la proporción específica en que se combinan ambos tipos de transporte.

El valor del transporte interior lo obtenemos de tres fuentes complementarias. De un lado, la base del impuesto que grava esta actividad en empresas con sede social en la provincia. De otro, los Datos Estadisticos de Norte, a fin de contabilizar los productos del transporte de las empresas domiciliadas en otras provincias. Finalmente, a partir de 1913 sustituimos la fuente fiscal por las memorias del Ferrocarril Cantábrico, aunque apenas hay diferencias entre ambas fuentes. Una vez estimado el producto del transporte terrestre, podemos estimar fácilmente el del marítimo; basta con indiciarlo en la proporción que relaciona ambos sistemas comerciales. Más adelante calcula Vandellós la renta del comercio «aplicando un porcentaje del 20 por ciento a los productos agricolas y a las minas y el 30 por ciento a los productos industriales.... ${ }^{42}$.

\subsection{SUBSECTOR FINANCIERO [S2]}

Limitamos el estudio a las instituciones financieras que están domiciliadas y realizan el grueso de sus actividades en la región. Ambas hipótesis son necesarias para poder atribuir geograficamente la riqueza generada y ambas son bastante plausibles ${ }^{43}$. En el período en que nos movemos la banca estaba poco especializada y su implantación era fundamentalmente local o regional; las operaciones en otras plazas quedaban a cargo de corresponsales. Para estimar

4 Vandellós (1977), p. 121.

42 lbid.

43 Roldan y García Delgado (1973), Tedde (1974a, 1974b), García Delgado (1984). 
el $\mathrm{VAB}$ recurrimos a las cuentas de pérdidas y ganancias de las sociedades, con lo que nos apartamos del método común al resto de los sectores. Los motivos de esta particularización hay que buscarlos en las peculiaridades del sector. El hecho de que esté perfectamente identificado facilita sobremanera la estimación directa, sin olvidar que resulta dificil derivar un concepto equivalente al de VPF.

\subsection{OTROS SERVICIOS $[\mathrm{S} 4]$}

Bajo este epigrafe tienen cabida administración pública, fuerza pública y ejército, servicios personales y domésticos, profesiones liberales, culto y clero. El principal problema radica en la valoración monetaria de servicios no-comercializables. La solución más socorrida consiste en estimar el VAB a partir de las cifras de empleo (Alcaide, 1976; Consejo de Economía Nacional, 1977). No han faltado críticas a esta forma de proceder ${ }^{44}$, si bien no hay que exage. rarlas. La estimación del producto a partir del empleo es satisfactoria, a condición de que el VAB per cápita sea correcto. El problema se centra, por tanto, en estimar las personas ocupadas y el VAB/empleo a aplicar.

En relación con el empleo, nos atenemos a los datos de la población activa censal, de la cual retenemos la clasificación por sexos. De otro lado, en términos de Contabilidad Nacional el VAB coincide con los gastos de personal, que incluyen salarios brutos y cargas de la Seguridad Social. A este respecto, desde el punto de vista documental hay dos etapas: antes y después de la guerra eu. ropea. Las estadísticas de esta última proceden del Ministerio de Trabajo y Previsión (1931): se trata de los salarios-hora percibidos por cinco categorias de trabajadores o profesiones-tipo en 1914, 1920, 1925 y 1930. El siguiente paso consiste en estimar el salario nominal semanal. A tal fin, se calcula el jornal. tipo como media ponderada del salario-hora por la duración de la jornada laboral, para lo cual se toma por defecto la estructura profesional de la población española ${ }^{45}$.

Para el periodo 1895.1913 el cálculo es más incierto, por falta de estadísticas de amplio espectro. Las investigaciones de Maluquer sugieren que hasta 1913 no hay cambios importantes en los precios y salarios ${ }^{46}$, por cuanto es posible indiciar la evolución de los salarios en el índice de precios, mejor conocido. Otra alternativa es usar estadísticas de empresas e instituciones, elevando a

\footnotetext{
(3) Schwartz (1977), p. 461.

45 Ministerio de Trabajo y Previsión (1931), pp. 27-28.

46 Maluquer (1987, p. 964; 1989).
} 
categoria provincial el indice resultante. Los problemas inherentes son de naturaleza estadistica, relacionados con la representatividad de la muestra.

Finalmente, hemos elaborado un indice de salarios nominales con base 1913-100. El índice de precios del periodo $1895-1913$ se supone representativo de la evolución salarial. En cuanto al periodo 1914-1930, los salarios de los años-corte se obtienen por interpolación, teniendo en cuenta la evolución del indice de salarios reales. El producto de salarios nominales y población activa mide el importe de los salarios brutos pagados por el subsector, por cuanto hay que suponer que se trata de un mínimo. En primer lugar, porque hablamos de salarios, no de retribuciones efectivas, que excluyen las retribuciones por razón de horas extraordinarias, pluses, complementos, etc. En segundo lugar, quedan excluidos los impuestos indirectos, las cargas a la seguridad social, etc.

\section{CONCLUSIONES}

El método descrito parece un camino alentador, con puntos fuertes y débiles, abierto a múltiples mejoras y no pocas criticas; es un punto de partida, no de llegada. En su haber cuenta con algunos activos: $1 .^{\circ}$ Utilización de métodos de análisis sectorial, propios de la historiografía económica cuantitativa. $2{ }^{\circ}$ Aplicación a los estudios de historia económica regional del rigor metodológico que se exige a los análisis sobre economia española o mundial. $33^{\circ}$ Homogeneización de todos los sectores en una magnitud común, el VAB, que posibilita la comparación entre provincias/regiones y sectores. $4 .^{\circ}$ Aproximación entre historia económica y economía aplicada, hasta formar un continuum en el que los procesos recientes adquieren sentido a la luz de la historia económica, y viceversa. $5 .^{\circ}$ Uso de técnicas cuantitativas que permiten contrastar determinadas hipótesis sobre el desarrollo económico regional.

En el debe anotamos, en primer lugar, la precariedad de las fuentes estadisticas provinciales. Se necesita hacer mucho trabajo de campo en empresas y en la depuración de datos. En segundo lugar, hay que mejorar las condiciones de aplicación de técnicas cuantitativas y empresariales al ámbito de la historia económica. Finalmente, sería conveniente empalmar el corto con el medio y largo plazo. En resumen, un camino prometedor pero algo escabroso todavía. 


\section{BIBLIOGRAFIA}

Alaejos, Luis (1922): «La pesca marítima en España en 1920: Provincia de Santander», Boletín de Pescas, n. ${ }^{03}$ 65-66-67, pp. 35-79.

- (1931): «La pesca maritima en el Puerto de Santanderm, Notas y Resúmenes, Ministerio de Fomento, pp. 22.23.

ALCAIDE, Julio (1976): «Una revisión urgente de la renta nacional de España en el siglo XX», Datos básicos para la bistoria financiera de España, 1850-1975. Madrid, Instituto de Estudios Fiscales, pp. 1.125-1.150.

Alvarez, Roberto (1986): «Evolución de la estructura económica regional de España en la historia», Siluación/1, Banco de Bilbao, pp. 5-61.

Banco BILbao VIzCAYA: Renta nacional de España y su distribución provincial.

CAmara de Comercio, Industria y Navegacion de Santander: Memoria sobre el movimiento comercial e industrial de la provincia de Santander.

Camara Oficial de Productores y Distribuidores de Electricidad (1935): Datos Estadísticos Técnicos de las Centrales Eléctricas Españolas.

Carmona, Xoan (1990): «Crisis y transformación de la base industrial gallega, 18501936», en Nadal, J. y Carreras, C., eds.: Pautas regionales de la industrialización española. Barcelona, Ariel, pp. 23-48.

CARreras, Albert (1984): «La producción industrial española, 1842-1941: construcción de un indice anual», Revista de Historia Económica, vol. 2, n. ${ }^{\circ} 1, \mathrm{pp} .127-157$.

- (1985): «Gasto nacional bruto y formación de capital en España, 1849-1958: Primer ensayo de estimación», en Martin Aceña, P. y Prados de la Escosura, L., eds.: La nueva historia económica de España. Madrid, Tecnos, pp. 17-51.

- (1989a): «La renta y la riqueza», en Carreras: Estadísticas históricas de España, siglos $X I X-X X$. Madrid, Fundación Banco Exterior, pp. 533-588.

- (1989b): Estadísticas bistóricas de España, siglos XIX.XX. Madrid, Fundación Banco Exterior.

- (1990): «Fuentes y datos para el análisis regional de la industrialización española», en Nadal y Carreras: Pautas regionales de la industrialización española. Barcelona, Ariel, pp. 3-20.

Chenery, H., y SYrQUin, M. (1978): La estructura del crecimiento económico. Un análisis para el período 1950-1970. Madrid, Tecnos.

Coll Martin, Sebastian (1989): «Estimación indirecta de una serie de renta nacional para España, 1874-1935. Primeros resultadosm, IV Congreso de la Asociación de Historia Económica. Alicante.

Coll y PUIG, J. Antonio (1891): Guia consultor e indicador de Santander y su provincia. Santander.

Compañia de los caminos de hIERro del nORTE de España (NORTE): Datos estadisticos.

COMPANIA DEL FERROCARRIL CANTABRICO: Memotias.

Consejo de ECONOM1a NACIONAL (1977): «[Introducción a] La renta nacional de Españaw, en Schwartz: El producto nacional de España en el siglo xx. Madrid, Instituto de Estudios Fiscales, pp. 217-231.

CONSEjo de MinerIa: Estadística Minera y Metalúrgica de España.

Cuadrado, J. Ramón, y Del Rio, Clemente (1990): «Los economistas y los servicios», Papeles de Economía Española, núm. 42, pp. 2-18.

D. G. DE ADUANAs: Praducción y circulación de azuicares, acbicoria, alcobol y cerveza. 
- Estadística general del comercio exterior de España (serie anual).

- Estadistica general del comercio de cabotaje de España (serie anual).

D. G. DE Agricultura, Industria y Comercio (1892): La ganadería en España. Auance sobre la riqueza pecuaria en 1891.

D. G. DE CONTRIBUCIONEs (1879): Estadística Administrativa de la Riqueza Territorial y Pecuaria.

_- Estadistica del impuesto sobre los transportes de viajeros y de mercancias par las vias terrestres y fluviales.

- Estadistica de la contribución sobre las utilidades de la riqueza mobiliaria.

D. G. DE RENTAS Públicas: Estadística sobre el consumo de luz de gas, electricidad y carburo de calcio.

Diputación Provincial de Santander (1929): La provincia de Santander en el último quinquenio, 1923-1928. Santander, Editorial de la Diputación Provincial.

ElectRA DE VIESGo: Memorias (varios años).

- (1981): Electra de Viesgo-75 años. Bilbao.

Fies: «Estimación del crecimiento del PIB por comunidades autónomas», Papeles de Economia Española. Anexos 1 a 6, correspondientes a los años 1986 a 1990.

__ (1990): «España: una economia de servicios», Papeles de Economía Española, n. ${ }^{\circ} 42$, Editorial.

FraIle, Pedro (1991): Industrialización y grupos de presión. La economia política de la protección en España, 1900-1950. Madrid, Alianza Editorial.

Frax, Esperanza (1981): Puertos y comercio de cabotaje en España, 1857-1934. Madrid, Banco de España.

Garcia Delgado, J. Luis (1984): «La industrialización española en el primer tercio del siglo XX», en Jover, J. M:, ed.: Historia de España Menéndez Pidal Madrid, EspasaCalpe, Tomo XXXVII, pp. 1-171.

Gerschenkron, Alexander (1968): «Notas sobre la tasa de desarrollo industrial italiana, en el período 1881.1913», en A. Gerschenkron: El atraso económico en su perspectiva bistórica. Barcelona, Ariel, pp. 79-96.

Gomez Mendoza, Antonio (1984): Ferrocarril y mercado interior en España (1874-1913). Cereales, harinas y vinos. Madrid, Banco de España.

(1985): Ferrocarril y mercado interior en España (1874-1913). Manufacturas textiles, minerales, combustibles y metales. Madrid, Banco de España.

(1986): «La industria de la construcción residencial: Madrid, 1820/1935m, Moneda y Crédito, nüm. 177, pp. 53-81.

- (1988): «Ferrocarril, abastecimientos y mercado nacional: Madrid, 1875-1931», en Bahamonde y Otero, eds.: La sociedad madrileña durante la Restauración, 1876-1931. Madrid.

Grupo de Estudios de Historia RuRaL, GEHR (1978): «Contribución al analisis histórico de la ganadería española, 1865-1929m, Agricultura y Sociedad, n. ${ }^{\circ}$, pp. 129-182 y n. ${ }^{\circ} 10$, pp. $105-169$.

- (1983): «Notas sobre la producción agraria española, 1891-1931», Revista de Historia Económica, vol. 1, n. ${ }^{\circ} 2$, pp. 185.252.

- 1985: «Evolución de la superficie cultivada de cereales y leguminosas en España, 1886.1935m, en Martín Aceña y Prados, eds.: La nueva bistoria económica de España. Madrid, Tecnos, pp. 52-70. 
- (1989a): «Sector agrario (hasta 1935)», en Carreras, A. ed.: Estadísticas históricas de España, siglos XIX-XX. Madrid, Fundación Banco Exterior, pp. 91-130.

- (1989b): «Las fuentes para el estudio de los montes públicos españoles, 1855 1935w, IV Congreso de la Asociación de Historia Económica. Alicante, pp. 277-297.

Instituto GeOGRAFICo Y Estadistico: Anuario(s) Estadístico(s) de Esparia.

- Censo(s) de la población española.

- Nomenclátor de las ciudades, villas, lugares, aldeas y demás entidades de población de España.

INTERVENCION GENERAL DE LA AdMINISTRACIÓN DEL Estado (1909): Estadistica de los Presupuestos Generales del Estado. Años 1890-91 a 1907.

Junta Consultiva Agronomica, JCA (1891): Avance estadístico sobre el cultivo cereal y de leguminosas a sociadas en España.

Junta Provincial de Abastos de Santander (1927): Memoria de la producción, consumo, comercio, precios, estadísticas de ganado y productos agro-pecuarios de la provincia de Santander.

MalUQuer, Jordi (1987): «Salarios y renta nacional (1913-1959)m, en Espina, Fina y Sáez, eds.: Estudios de economía del trabajo en España. II. Ministerio de Trabajo y Seguridad Social, pp. 959-978.

- (1989): «Precios, salarios y beneficios. La distribución funcional de la renta», en Carreras, ed.: Estadísticas bistóricas de España, siglos XIX-XX. Madrid, Fundación Banco Exterior, pp. 495.532.

Ministerio de Agricultura, Industria, Comercio y Obras Públicas (1901): Estadística de la industria eléctrica en España en fin de 1901.

- (1902): Noticias estadísticas sobre la producción agricola española.

(1905a): Pastos y prados.

- (1905b): Memoria sobre el estado de la industria en la provincia de Santander.

Ministerio de Agricultura, Industria y Comercio. D. G. DE Agricultura (1932): Anuario Estadístico de las Producciones Agricolas.

Ministerio de Economia Nacional. D. G. DE Agricultura (1929, 1930 y 1933): Anuario Estadistico de las Producciones Agrícolas.

Ministerio DE Fomento (1910): Estadística de la industria eléctrica en España en fin de 1910.

- (1911): Estadistica comercial e industrial de la provincia de Santander.

- (1930): Momento de la industria española.

Ministerio de Fomento. D. G. DE Agricultura, Minas y Montes (1913): Avance estadistico de la riqueza que en España representa la producción media anual de árboles y arbustos frutales $(y)$ tubérculos, raices $y$ bulbos.

- (1914a): Avance estadistico de la riqueza que en España representa la producción media anual de las plantas borticolas y plantas industriales.

- (1914b): Avance estadístico de la riqueza que en España representa la producción media anual de las pastos, prados y algunos aprovechamientos $(y)$ pequerias industrias zoógenas anexas.

- (1915): Avance estadistico de la riqueza que en España representa la producción media anual de cereales, leguminosas, vid y olivo (y) aprovechamientos diversos derivados de estos cultivos.

- (1920): Estudio de la ganadería en España.

(1923): Avance estadístico de la producción agrícola en España.

Ministerio de Fomento. D. G. DE Agricultura, Industria y Comercio: Boletín Semanal de Estadistica y Mercados. 
- Boletín de Agricultura Técnica y Económica.

Ministerio DE HACIENDA (1897): Estadistica Tributaria de España. Año económico de 1894-95.

(1900): «Memorias sobre la industria fabril redactadas por los ingenieros al servicio de la Hacienda Públicas, Gaceta de Madrid, núm. 117, de 27 de abril.

- (1960): «Dictamen de la Comisión nombrada por R.O. de 9 de enero de 1929 para el estudio de la implantación del patrón de orow, Información Comercial Española, febrero, pp. 51.83.

Ministerio de Hacienda. D. G. de Propiedades y Contribucion Territorial (1931): Memoria del año 1928.

Ministerio de Hacienda. Servicio del Catastro de la Riqueza Urbana (1918): Estado de los trabajos y resultados obtenidos basta 31 de diciembre de 1917.

- (1921): Estado de los trabajos y resultados obtenidos basta 31 de marzo de 1919.

- (1922): Estado de los trabajos y resultados obteridos basta 31 de marzo de 1921.

Ministerio de Industria y Comercio. Subsecretaria de LA Marina Civil (1933): Estadísiica de pesca.

Ministerio de Marina. D. G. de Navegacion y Pesca Maritima: Anuatio de Pesca y Estadístico.

Ministerio de Trabajo y Prevision (1931): Estadística de salarios y jomadas de trabajo, referida al periodo 1914-1930.

Molinas, César, y Prados, Leandro (1989): «Was Spain Different? Spanish Historical Backwardnes Revisited». Explorations in Economic History, vol. 26, n. ${ }^{\circ} 4$, pp. 385-402.

MuNoz, Cándido (1991): Introducción a la economía aplicada. Madrid, Biblioteca de economia. Espasa-Calpe.

Nadal, Jordi; Carreras, Albert, y Sudria, Carles (1987), eds.: La economia española en el siglo $x x$. Una perspectiva histórica. Barcelona, Ariel.

NADAL, Jordi, y CARRERAS, Albert (1990): Pautas regionales de la industrialización española. Barcelona, Ariel.

ORTEGA, José (1986): Cantabria 1886-1986: formación y desarrollo de una economía maderna. Santander, Estudio.

- 1990: «La industrialización en Cantabria (1844-1944). Génesis de una industria especializada», en Nadal y Carreras, eds.: Pautas regionales de la industrialización española. Barcelona, Ariel, pp. 79.105.

Perez, Patricio (1990): «Economia y calidad de vida en Cantabria durante el primer tercio del siglo XX», XV Simposio de Análisis Económico. Barcelona, pp. 117-126.

- (1991): Crecimiento económico y cambio estructural de Cantabria durante el primer tercio del siglo $\times x$. Tesis doctoral, Universidad de Cantabria.

- (1992): "La industria de bienes de consumo en la provincia de Santander, 1895. 1930w, VI Simposio de Historia Económica. Barcelona, pp. 182-196.

Pinilla, Vicente (1990): La producción agraria en Aragón (1850-1935). Tesis doctoral, Universidad de Zaragoza.

Prados, Leandro (1982): Comercio exterior y crecimiento económico en España, 1826-1913: cendencias a largo plazo. Madrid, Banco de España.

(1986): «Una serie anual del comercio exterior español (1821-1913)», Revista de Historia Económica, vol. 4, n..$^{\circ}$, pp. 103-150.

- (1988): De imperio a nación. Crecimiento y atraso económico en España (17801930). Madrid, Alianza Editorial. 
Pujol, Josep (1988): Les transformacions del sector agrari catala entre la crisi finisecular $i$ la Guerra Civil. Tesis doctoral, Universidad Autónoma de Barcelona.

Rojo, L. Angel (1979): Renta, precios y balanza de pagos. Madrid, Alianza Editorial, 5." edición.

RoldAn, Santiago, y GARCI Delgado, J. Luis (1973): La formación de la sociedad capilalista en España, 1914-1920. Madrid, Confederación Española de Cajas de Ahorros.

SANCHEZ-ALBORNOZ, Nicolás (1985): La modernización económica de España. 1830-1930. Madrid, Alianza Editorial.

SCHwartz, Pedro (1977): «El producto interior bruto en España de 1940 a 1960», en Schwartz: El producto nacional de España en el siglo Xx. Madrid, Instituto de Estudios Fiscales, pp. 443-592.

Seccion Agronomica de Santander: Memorias Reglamentarias de 1889, 1890, 1900 y 1908. Originales manuscritos.

TAfUNELL, Xavier (1988): La construcción residencial en el crecimiento económico en Barcelona (1854-1879). Tesis doctoral, Universidad Autónoma de Barcelona.

- (1989): «La construcción», en Carreras: Estadísticas bistóricas de España, siglos XIX.XX. Madrid, Fundación Banco Exterior, pp. 251-267.

TEDDE, Pedro (1974a): «La banca privada española durante la Restauración (1874. 1914)», en Schwartz, P., ed.: La Banca española en la Restauración. Política y finanzas. Madrid, Banco de España, Tomo I, pp. 217-455.

- (1974b): «Agregación regional de las principales magnitudes bancarias (1874. 1914)», en Schwartz (ed.): La banca española de la Restauración. Datos para una bistoria económica. Madrid, 1974. Tomo II, Banco de España, pp. 491-525.

TENA, Antonio (1985): «Una reconstrucción del comercio exterior español, 1914-1935: la rectificación de las estadisticas oficiales", Revista de Historia Económica, vol. 3, n. ${ }^{\circ}$ 1, pp. $77-119$.

VANDELLOS, Josep (1977): «La riqueza y la renta de la peninsula ibérica», en Schwartz: El producto nacional de España en el siglo XX. Madrid, Instituto de Estudios Fiscales, pp. $91-138$. 